\title{
Effetto della restrizione alimentare di dieci ore al giorno su peso corporeo, pressione arteriosa e lipidi aterogeni in pazienti affetti da sindrome metabolica
}

\author{
Angelo Cignarelli ${ }^{1}$. Valentina A. Genchi ${ }^{1}$
}

Accettato: 15 aprile 2020 / Pubblicato online: 28 agosto 2020

(c) The Author(s) 2020

Commento a:

Ten-hour time-restricted eating reduces weight, blood pressure, and atherogenic lipids in patients with metabolic syndrome.

M.J. Wilkinson, E.N. Manoogian, A. Zadourian, H. Lo, S. Fakhouri, A. Shoghi, X. Wang, J.G. Fleischer, S. Navlakha, S. Panda, P.R. Taub.

Cell Metab (2020) 31(1):92-104.e5

Diversi studi in vivo su modelli animali di obesità e di sindrome metabolica (SM) hanno dimostrato come la restrizione temporale dell'alimentazione (time-restricted eating, TRE) sia in grado di determinare un significativo calo ponderale con una riduzione dell'adiposità e un miglioramento metabolico in termini di ripristino della tolleranza glucidica e riduzione dei livelli di colesterolo e trigliceridi [1,2]. Tuttavia, ad oggi non ci sono evidenze che documentino gli effetti metabolici di un regime alimentare basato sul TRE in pazienti affetti da SM.

L'obiettivo di questo studio è stato quello di valutare in trentacinque pazienti con SM gli effetti di un programma di TRE sul metabolismo, sul peso corporeo e sulla distribuzione della massa grassa. L'intervento è stato condotto per 12 settimane con l'esclusiva richiesta di alimentarsi in un arco temporale limitato giornaliero di 10 ore, ed è stato monitorato mediante il supporto informatico di una app (myCircadianClock, mCC app) con lo scopo di annotare gli orari dei pasti, l'intake calorico e il ciclo sonno-veglia. I pazienti sono stati sottoposti, prima e dopo l'intervento, a valutazione dei parametri antropometrici (peso, BMI, circonfe-

$凶$ A. Cignarelli

angelo.cignarelli@gmail.com

1 Sezione di Medicina Interna, Endocrinologia, Andrologia e Malattie Metaboliche, Dipartimento di Emergenza e dei Trapianti di Organo, Università di Bari “Aldo Moro”, Bari, Italia renza vita), dei livelli circolanti dei lipidi (colesterolo totale, LDL, non-HDL, trigliceridi), dei marcatori di infiammazione (PCR) e di parametri metabolici (glicemia, HbAlc, insulinemia, HOMA-IR).

La coorte arruolata ha mostrato una buona aderenza al trattamento con un miglioramento della qualità del sonno e del riposo. Il programma TRE si è associato a un significativo calo ponderale $(-3 \%)$, a una riduzione del BMI $(-3 \%)$, della circonferenza vita $(-4 \%)$ e del grasso viscerale $(-3 \%)$. Inoltre, è stato osservato un decremento nei livelli di colesterolo totale $(-7 \%)$, colesterolo LDL $(-11 \%)$ dopo l'intervento, così come una significativa riduzione della pressione arteriosa sistolica e diastolica $(-4 \mathrm{e}-8 \%$, rispettivamente). È interessante notare come gran parte dei pazienti fosse già in terapia farmacologica (79 e 63\% in terapia con statina e anti-ipertensivi, rispettivamente) suggerendo come i benefici ottenuti con il TRE sia additivo all'effetto dei farmaci; inoltre, va rimarcato come gli effetti delle variazioni lipidiche e pressorie siano risultate statisticamente indipendenti dalle variazioni ponderali.

Il trattamento non ha comportato evidenti variazioni glicemiche, sebbene nei soggetti che al basale presentavano livelli di glucosio a digiuno più elevati abbiano mostrato un miglioramento della glicemia e dei livelli di HbA1c. Infine, al termine dello studio l'introito calorico è risultato ridotto (involontariamente) di circa il 10\%.

In questo interessante lavoro, gli autori dimostrano come restringere la finestra temporale in cui alimentarsi a 10 ore al giorno in pazienti con SM sia in grado di favorire un significativo calo di peso e un miglioramento della distribuzione del grasso corporeo. In particolare, i pazienti sottoposti al regime TRE mostrano un miglioramento del profilo lipidico e della pressione arteriosa apparentemente indipendenti dalla variazione ponderale suggerendo, quindi, come il programma alimentare di per sé possa favorire un miglioramento del 
quadro metabolico complessivo. Pertanto, questi dati innovativi aprono nuove frontiere per la cura della SM, indicando come un intervento a costo zero, ovvero la restrizione dell'intervallo temporale in cui alimentarsi, sia in grado anche di potenziare l'efficacia della terapia farmacologica in uso.

Restano da chiarire alcuni punti salienti dello studio come, ad esempio, il potenziale contributo del miglioramento del ritmo circadiano e della qualità del sonno sull'effetto della terapia farmacologica, vista la relazione nota tra farmaci e ritmo sonno/veglia [3], così come è altresì importante comprendere se la riduzione dell'introito calorico a cui si assiste al termine del trattamento possa essere il risultato di variazioni ormonali dell'asse intestino-cervello e dei livelli dei principali ormoni anoressizzanti (PYY, leptina, GLP-1) conseguenti ad una variazione del bioritmo [4].

Funding Note Open access funding provided by Università degli Studi di Bari Aldo Moro within the CRUI-CARE Agreement.

Nota della casa editrice Springer Nature rimane neutrale in riguardo alle rivendicazioni giurisdizionali nelle mappe pubblicate e nelle affiliazioni istituzionali.

Open Access This article is licensed under a Creative Commons Attribution 4.0 International License, which permits use, sharing, adap- tation, distribution and reproduction in any medium or format, as long as you give appropriate credit to the original author(s) and the source, provide a link to the Creative Commons licence, and indicate if changes were made. The images or other third party material in this article are included in the article's Creative Commons licence, unless indicated otherwise in a credit line to the material. If material is not included in the article's Creative Commons licence and your intended use is not permitted by statutory regulation or exceeds the permitted use, you will need to obtain permission directly from the copyright holder. To view a copy of this licence, visit http://creativecommons.org/licenses/by/4.0/.

\section{Bibliografia}

1. Chung H, Chou W, Sears DD et al (2016) Time-restricted feeding improves insulin resistance and hepatic steatosis in a mouse model of postmenopausal obesity. Metabolism 65:1743-1754

2. Chaix A, Zarrinpar A, Miu P, Panda S (2014) Time-restricted feeding is a preventative and therapeutic intervention against diverse nutritional challenges. Cell Metab 20:991-1005

3. Cederroth CR, Albrecht U, Bass J et al (2019) Medicine in the fourth dimension. Cell Metab 30:238-250

4. Westerterp-Plantenga MS (2016) Sleep, circadian rhythm and body weight: Parallel developments. Proc Nutr Soc 75:431-439 